\title{
Electrothermal Limitations on the Current Density of High-Frequency Bipolar Transistors
}

\author{
Nebojša Nenadović, Student Member, IEEE, Lis K. Nanver, Member, IEEE, and Jan W. Slotboom, Member, IEEE
}

\begin{abstract}
In this paper, electrothermal consequences of downscaling bipolar transistors, reducing the emitter resistance and implementing substrate modifications are examined by means of electrical measurements, numerical simulations and analytical calculations. A formulation is given for the optimum current density that can be run through the device and still maintain both sufficient transconductance and thermal stability. This expression sets a theoretical limit on the current density and therefore also on the speed of the given technology node. Particularly the lowering of the emitter resistivity is a trade-off between transconductance and thermal stability, and the optimum choice can be estimated from these results along with the maximum emitter area that will allow unconditional thermal stability.
\end{abstract}

Index Terms-Bipolar transistors, emitter resistance, high-frequency (HF) technologies, silicon-on-glass, thermal instability.

\section{INTRODUCTION}

$\mathbf{T}$ O INCREASE the speed and overall performance of highfrequency (HF) silicon bipolar devices and circuits, the microelectronics industry is continually investing in the downscaling of devices fabricated on the surface of silicon wafers. The present trends for emitter area downscaling and current densities at maximum cut-off frequency $f_{T, \max }$ are displayed in Fig. 1, where it is clear that there is an almost linear relationship between the current density $J_{C}$ at $f_{T, \max }$ and the $f_{T, \max }$ itself. Thus, to achieve the higher speed, higher current densities are being implemented [1]-[8]. The achievable currents are directly limited by the emitter contact series resistance, which is inversely proportional to the emitter area. In bipolar devices it is therefore particularly important to develop techniques that reduce the specific emitter resistivity [9]. However, the trade-offs for high collector current and low emitter resistance are twofold. For the first, the power dissipation and corresponding self-heating in the device is high, and second, in the absence of series resistors the electrothermal feedback is stronger and the device is more prone to thermal breakdown [10].

In this paper, the trade off between device speed and thermal stability is evaluated quantitatively by establishing a set of simplified analytical formulas for the collector current/thermal breakdown relationships. The presented calculations show that the electrothermal limitations on the current density, and thus also the device speed, can become quite severe in future

Manuscript received June 14, 2004; revised October 11, 2004. The review of this paper was arranged by Editor V.R. Ramgopal.

The authors are with the ECTM Laboratory, Delft Institute of Microelectronics (DIMES), Delft University of Technology, Delft 2600 GB, The Netherlands (e-mail: n.nenadovic@dimes.tudelft.nl).

Digital Object Identifier 10.1109/TED.2004.839754 device generations. The situation is particularly critical because of the need in HF circuits to eliminate both electrical device and circuit parasitics. Many substrate modification techniques are being introduced to improve transistor speed, improve the quality of integrated passives and reduce crosstalk. These include shallow and deep trench isolation, silicon-on-insulator (SOI) processes and substrate transfer [11], [12]. In all these methods, the electrically conductive silicon is replaced by electrical insulators that, unfortunately, also are very good thermal insulators and the thermal resistance of the devices can become forbiddingly high. The thermal resistance of several different device structures is estimated by simulations and the associated thermal instabilities are evaluated by correlating to measurements of bulk-silicon and silicon-on-glass transistors. The silicon-on-glass NPN BJTs are thoroughly described and experimentally investigated in [13]. Together with data recently published on today's most advanced silicon devices, the experimental results on the silicon-on-glass transistors are used to support the analysis and calculations.

\section{Self-Heating AND Thermal BREaKdown In BJTs}

In the following, very simple electrothermal relationships are applied to arrive at an equally simple analytical formulation that describes how the current through a device is limited by self-heating and/or emitter resistance. When the structure of the device is well known and the thermal resistance can be accurately determined, it is possible to extend the present analysis by using more accurate but also more complicated formulations of the thermal resistance [14], [15]. Here we limit ourselves to first-order approximations. Hence, the thermal resistance $R_{\mathrm{TH}}$ of a bulk bipolar transistor is related to the emitter area $A_{E}$ and the thermal conductivity $k_{\text {bulk }}$ of the bulk material by the simple equation [14]

$$
R_{\mathrm{TH}}=\frac{1}{4 k_{\mathrm{bulk}} \sqrt{A_{E}}} .
$$

The power $P$ dissipated in the device is related to the power density $p$ as $P=A_{E} p=A_{E} J_{C} V_{\mathrm{CE}}$, where $J_{C}$ is the collector current density and $V_{\mathrm{CE}}$ the collector-emitter voltage. Thus the temperature increase above ambient $\Delta T$ is related to $J_{C}$ as

$$
\Delta T=R_{\mathrm{TH}} P=R_{\mathrm{TH}} A_{E} p=\frac{p \sqrt{A_{E}}}{4 k_{\mathrm{bulk}}}=\frac{J_{C} V_{\mathrm{CE}} \sqrt{A_{E}}}{4 k_{\mathrm{bulk}}} .
$$

This equation is used to calculate the $\Delta T$ at $f_{T, \max }$ in Fig. 1. For a constant power density, a reduction in device size would reduce the self-heating. However, due to the trend to increase power density to achieve higher $f_{T}$ as shown in Fig. 1, the 

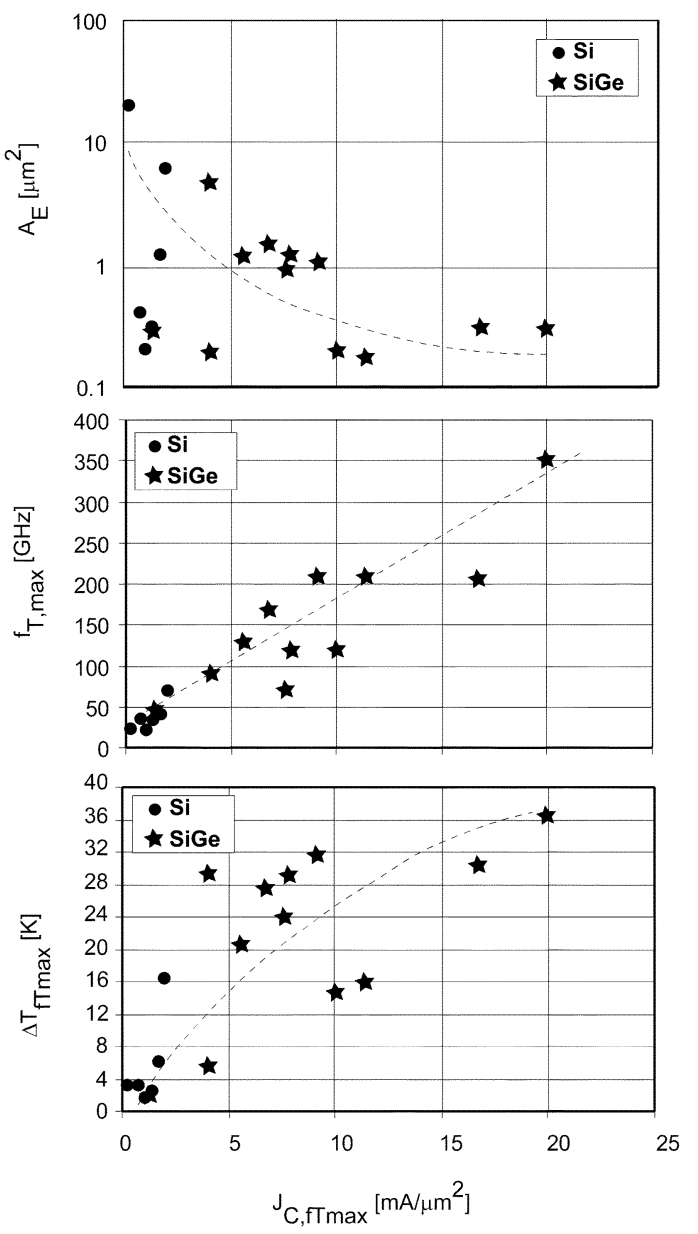

Fig. 1. Emitter area $A_{E}$, peak cut-off frequency $f_{T, \max }$ and approximated temperature increase at $f_{T, \max }, \Delta T_{f T \text { max }}$, versus current density $J_{C, f T \max }$ at $f_{T, \max }$ for recently published $\mathrm{Si}$ and $\mathrm{SiGe}$ transistors [1]-[8].

self-heating increases despite reduction in the device size. In addition, for most modern devices the $R_{\mathrm{TH}}$ cannot be approximated by such simple formulations, since for example effects of shallow and deep trench isolation, and buried oxide have to be taken into account [14]. In order to give an illustration of the effect of the substrate modifications and packaging on the self-heating, two-dimensional numerical thermal simulations are performed in Femlab [16] and the results are shown in Fig. 2. The bulk-silicon technology in Fig. 2A is compared to the bulk-silicon technology with deep trenches in Fig. 2B, SOI technology without and with trenches in Fig. 2C and Fig. 2D respectively, and silicon-on-glass technology [11] in E. It can be seen that as the electrical isolation gets better, the thermal resistance gets undesirably high. Good heat sinking is often implemented in the packaging. This is particularly effective if the bulk silicon is replaced by a better thermal conductor as in F where a silicon-on-glass technology with copper heat-sink is represented. Here the thermal resistance becomes lower than the bulk silicon value. Such a technology is successfully implemented in experimental silicon-on-glass BJTs [12] and silicon-on-glass power MOSFETs [17]. Although the simulated structures are very simplified and three-dimensional effects are not included, the trend in self-heating is overly clear and illustrates the enormous significance of the device geometry
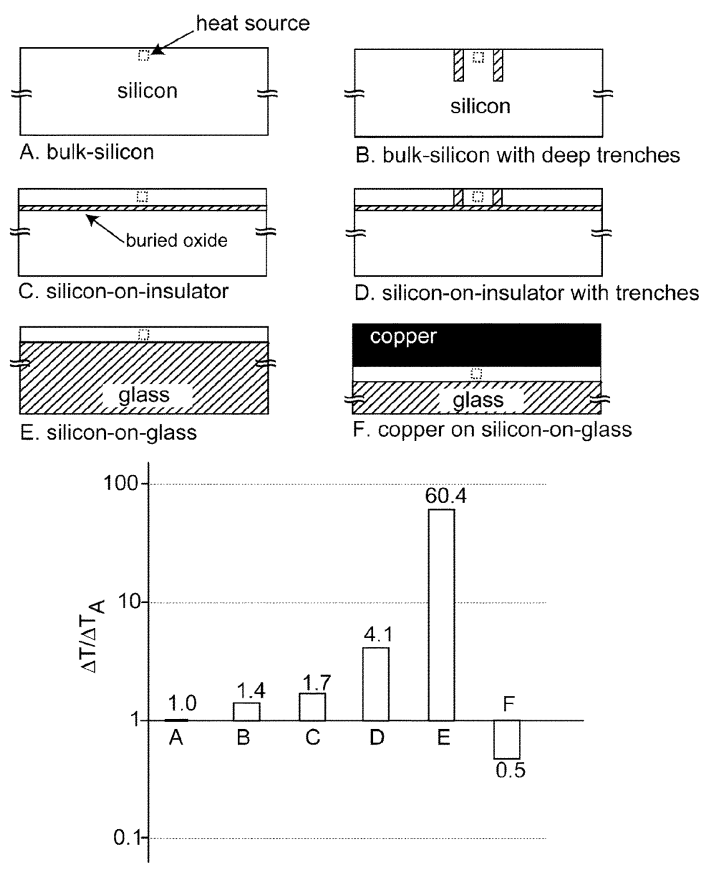

Fig. 2. Numerically simulated temperature increase with respect to ambient for different technologies. The temperatures are calculated for the same input power and normalized to $\Delta T$ of $\mathrm{A}$.

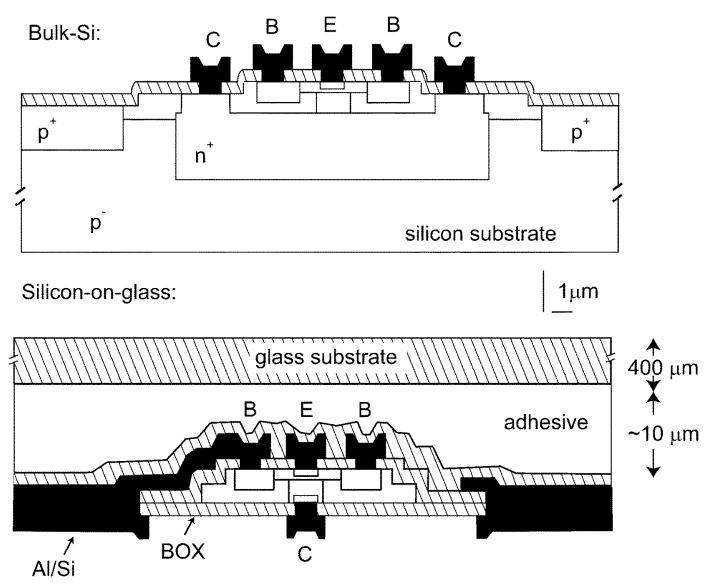

Fig. 3. Schematic cross sections of experimentally studied bulk-silicon and silicon-on-glass NPN BJTs from the same processing line. For more details on the silicon-on-glass processing and silicon-on-glass NPN BJT architecture refer to [13].

and surrounding on the electrothermal limitations described below.

The electrothermal interaction in bipolar transistors can lead to thermal instability [18], [19] and dynamic temperature-current positive feedback can even result in destructive thermal breakdown [20]. Experimental Gummel plots of the bulk-silicon and silicon-on-glass NPN BJTs [13] with schematic cross sections given in Fig. 3 and an emitter area $A_{E}$ of $20 \times 1 \mu \mathrm{m}^{2}$ are shown in Fig. 4(a). In the silicon-on-glass transistor the thermal breakdown is seen as a sudden increase in the collector and base current. By assuming that the collector current ideality factor is 

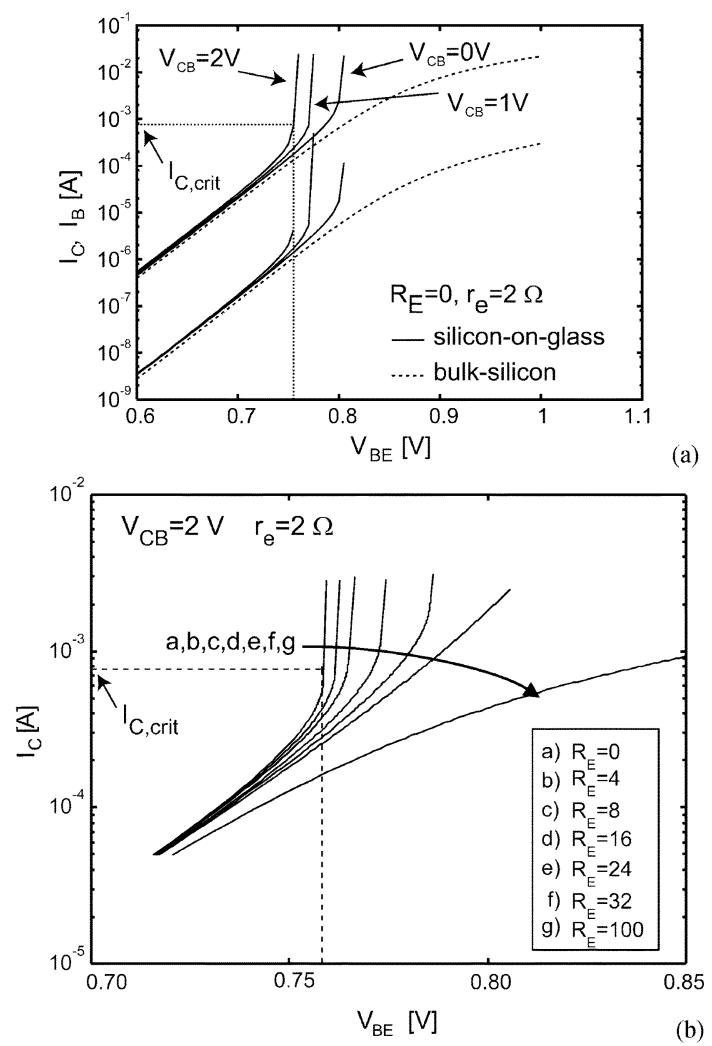

Fig. 4. Measurements of the transistors shown in Fig. 3. (a) Gummel plots of a bulk-silicon (dashed lines) and silicon-on-glass devices (solid lines), and (b) Gummel plots of the silicon-on-glass device for several externally added emitter series resistors $R_{E}$. Emitter area is $20 \times 1 \mu \mathrm{m}^{2}$.

1 , the collector current density at the onset of thermal breakdown, $J_{C, \text { crit }}$, can be calculated by [18]

$$
J_{C, \text { crit }}=\frac{I_{C, \text { crit }}}{A_{E}}=\frac{\frac{V_{T}}{A_{E}}}{|\varphi| V_{\mathrm{CE}} R_{\mathrm{TH}}-R_{\Sigma}}
$$

where $V_{T}=k T / q(\approx 26 \mathrm{mV})$ is the thermal voltage, $\varphi$ the base-emitter voltage temperature coefficient $(\approx-1 \mathrm{mV} / \mathrm{K})$ and $R_{\Sigma}=R_{E}+r_{e}$ the equivalent electrical emitter resistance, including the internal $r_{e}$ and externally applied $R_{E}$ resistance terms. The internal resistance $r_{e}$ is usually expressed in terms of the specific emitter resistivity $r_{\mathrm{es}}=r_{e} A_{E}$ in $\Omega \mu \mathrm{m}^{2}$, which is a technology parameter. From device measurements, the thermal resistance is determined to be about 10000 and $300 \mathrm{~K} / \mathrm{W}$ for the silicon-on-glass and bulk-silicon device, respectively. For $V_{\mathrm{CB}}=2 \mathrm{~V}$ and $R_{\Sigma}=2 \Omega$ this gives $I_{C, \text { crit }} \approx 1 \mathrm{~mA}$ for the silicon-on-glass device and unconditional stability for the bulk-silicon transistor since $|\varphi| V_{\mathrm{CE}} R_{\mathrm{TH} \text {,bulk }}-R_{\Sigma}<0$.

The series resistance $R_{\Sigma}$ introduces negative feedback, which thermally stabilizes devices [10]. This effect is illustrated in Fig. 4(b), in which experimental Gummel plots of the silicon-on-glass device are shown for different $R_{E} \mathrm{~s}$. The voltage drop across the emitter series resistance also reduces the current-normalized transconductance $g_{m}$

$$
\frac{g_{m}}{I_{C}}=\frac{1}{V_{T}+I_{C} R_{\Sigma}} .
$$

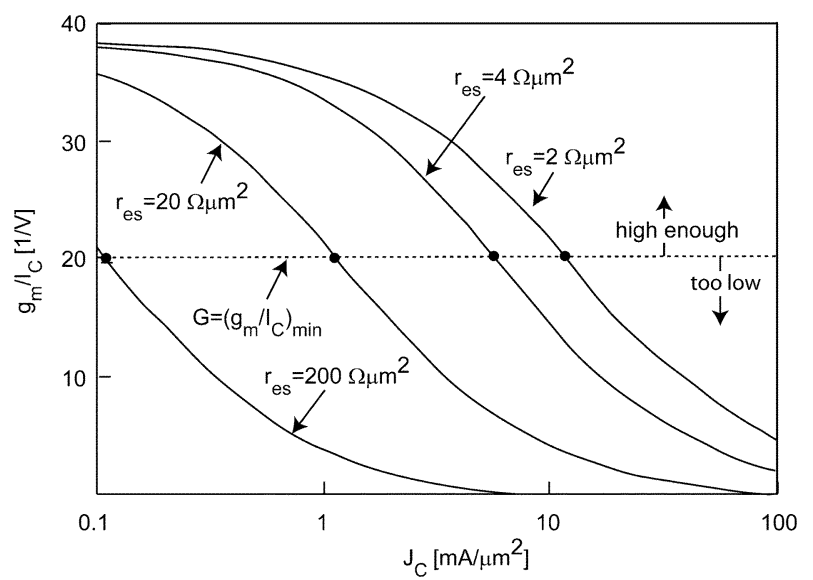

Fig. 5. Current-normalized transconductance versus collector current density for different values of specific emitter resistance. External emitter series resistance $R_{E}$ is neglected. $J_{C \text {, max }}$ is highlighted by the filled circles.

For most applications a $\left(g_{m} / I_{C}\right)$ value of about half the maximum value (for $R_{\Sigma}=0$ ) is still useful. Therefore the quantity

$$
G=\left(\frac{g_{m}}{I_{C}}\right)_{\min }=\frac{1}{\left(2 V_{T}\right)} \approx 20 \mathrm{~V}^{-1}
$$

is introduced here as a lower limit on the current-normalized transconductance [21]. The maximum current density at which $\left(g_{m} / I_{C}\right)$ is above or equal to $20 \mathrm{~V}^{-1}$ for a given $R_{\Sigma}$, is evaluated from (4) and (5) as

$$
J_{C, \max }=\frac{1-V_{T} G}{A_{E} R_{\Sigma} G} .
$$

From Fig. 5 it is clear that as the series resistance gets higher, the $J_{C, \max }$ gets lower. Therefore, for operation at very high current densities, which is typical for modern HF devices, the series resistance must be minimized [22]. For instance, to reach the highest achieved $f_{T, \max }$ of $350 \mathrm{GHz}$, the $J_{C, f T \text { max }}$ needs to be as high as $20 \mathrm{~mA} / \mu \mathrm{m}^{2}$. Even in the absence of any external emitter resistance, i.e., for $R_{E}=0$, such a high current density will limit $\left(g_{m} / I_{C}\right)$ to below $20 \mathrm{~V}^{-1}$ if the technology advances do not allow $r_{\mathrm{es}}$ to be reduced to $1.2 \Omega \mu \mathrm{m}^{2}$. For such a low value of $r_{\text {es }}$, the negative feedback in the emitter is almost completely suppressed, which directly means that the electrothermal feedback is significantly increased.

\section{Analytical Formulations of $A_{E \text {,crit }}$ AND $J_{C \text {,opt }}$}

In the analysis in this section it is assumed that the external emitter resistance $R_{E}$ is negligible, thus $R_{\Sigma}=r_{e}=r_{\mathrm{es}} / A_{E}$. According to (3), the thermal instability occurs only if $|\varphi| V_{\mathrm{CE}} R_{\mathrm{TH}}-R_{\Sigma}>0$. Therefore, with the expression for $R_{\mathrm{TH}}$ given by (1), the maximum emitter area $A_{E, \text { crit }}$ for which the device is unconditionally thermally stable for given $r_{\mathrm{es}}$, $k_{\text {bulk }}$ and $V_{\mathrm{CE}}$ is expressed by

$$
A_{E, \text { crit }}=\frac{16}{\varphi^{2} V_{\mathrm{CE}}^{2}} k_{\mathrm{bulk}}^{2} r_{\mathrm{es}}^{2}
$$

This means that a device with $A_{E} \leq A_{E, \text { crit }}$ cannot be driven into thermal breakdown. Otherwise, for $A_{E}>A_{E \text {,crit }}$ the thermal breakdown occurs for $J_{C, \text { crit }}$ defined by (3). 
From (3) and (6) it is shown that while lowering the emitter resistance increases $J_{C \text {, max }}$, it will decrease $J_{C \text {,crit }}$. Therefore, there is an $r_{\mathrm{es}}$ for which $J_{C, \max }=J_{C \text {,crit }}$. This defines an optimum value for the specific emitter resistivity $r_{\text {es,opt }}$, which optimizes the allowable current density $J_{C \text {,opt }}$ of a bipolar transistor for given $A_{E}, V_{\mathrm{CE}}$, and $R_{\mathrm{TH}}$

$$
r_{\mathrm{es}, \mathrm{opt}}=A_{E} V_{\mathrm{CE}} R_{\mathrm{TH}} \varphi\left(1-G V_{T}\right)
$$

and

$$
J_{C, \mathrm{opt}}=\frac{1}{A_{E} G \varphi V_{\mathrm{CE}} R_{\mathrm{TH}}} .
$$

The $J_{C \text {,opt }}$ is the optimum current density of a single-finger HF bipolar transistor made in a given technology node. Keeping emitter area constant while reducing the specific emitter resistivity below $r_{\text {es,opt }}$ is not beneficial for the speed of BJTs: for $r_{\text {es }} \leq r_{\text {es,opt }}$ the current is limited by the thermal instability to $J_{C \text {,crit }} \leq J_{C \text {,opt }}$, while for $r_{\text {es }} \geq r_{\text {es,opt }}$ the current is limited by $\left(g_{m} / I_{C}\right)_{\min }$ to $J_{C, \max } \leq J_{C \text {,opt }}$.

In a multifinger device, the mutual thermal coupling resistance $R_{M}$ between the neighboring device fingers is very important for the thermal stability. Moreover, in such devices, the $J_{C \text {,crit }}$ depends on the type of biasing [23]. If the thermal interaction between more distant fingers is negligible, (9) can be written to include the effect of $R_{M}$

$$
J_{C, \mathrm{opt}}=\frac{1}{A_{E} G \varphi V_{\mathrm{CE}}\left(R_{\mathrm{TH}} \pm R_{M}\right)}
$$

where + and - corresponds to voltage-controlled and currentcontrolled biasing, respectively.

\section{Discussion}

In this section, the simple analytical formulations established in (1) to (10) are applied for a number of different device and technology parameters. First, $r_{\mathrm{es}}$ is set to $10 \Omega \mu \mathrm{m}^{2}, V_{\mathrm{CE}}$ to $2 \mathrm{~V}$ and $\varphi$ to $-1 \mathrm{mV} / \mathrm{K}$, and the critical area expressed by (7) is plotted in Fig. 6(a) versus the thermal conductivity of the substrate $k_{\text {bulk }}$. A device with an emitter area smaller than $9 \mu \mathrm{m}^{2}$ is unconditionally stable for bulk-silicon technology. On the other hand, if a device is completely surrounded by glass, the critical area is almost four to five orders of magnitude smaller. The figure shows that a small device, which is theoretically not prone to thermal breakdown if made in bulk-silicon technology, can experience instability problems if made in the trench-isolated, SOI or especially silicon-on-glass technology. Also, replacing the silicon wafer with a more thermally conducting substrate like copper [24], lowers the thermal resistance and can be beneficial for thermal stability of large transistors.

In Fig. 6(b) $A_{E \text {,crit }}$ is plotted as a function of $r_{\mathrm{es}}$ for devices surrounded by either glass or silicon. For the measured silicon-on-glass and bulk-silicon devices from Figs. 3 and 4 the $r_{\mathrm{es}}$ is $40 \Omega \mu \mathrm{m}^{2}$, which corresponds to $A_{E \text {, crit }}$ (silicon) $=144 \mu \mathrm{m}^{2}$ and $A_{E \text {,crit }}$ (glass) $=0.007 \mu \mathrm{m}^{2}$. Therefore, the device with $A_{E}=20 \mu \mathrm{m}^{2}$ is unconditionally stable in bulk-silicon technology, while it suffers from the thermal breakdown if made in silicon-on-glass technology. For the most advanced device from Fig. 1 with $A_{E}=0.3 \mu \mathrm{m}^{2}, f_{T, \max }=350 \mathrm{GHz}, J_{C, f T \max }=$
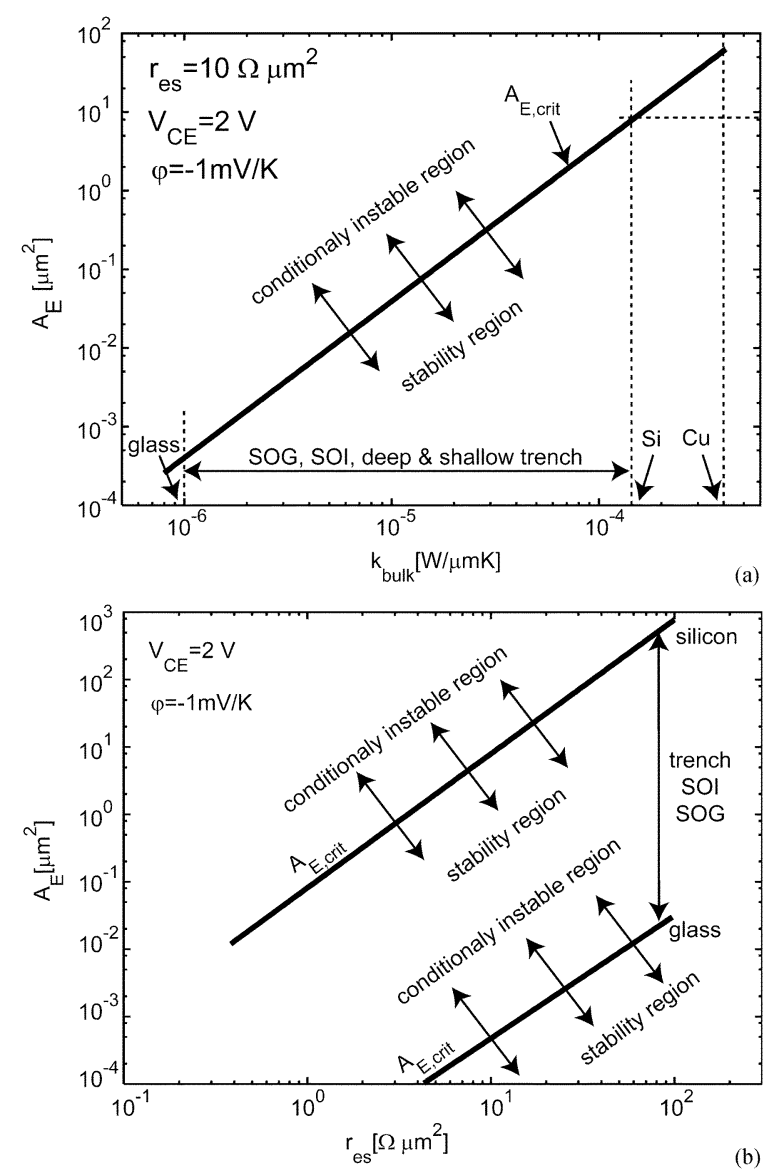

Fig. 6. Maximum emitter area for which a bipolar transistor is thermally stable (a) versus thermal conductivity of the substrate for a fixed specific emitter resistivity and (b) versus specific emitter resistivity for silicon and glass substrate materials.

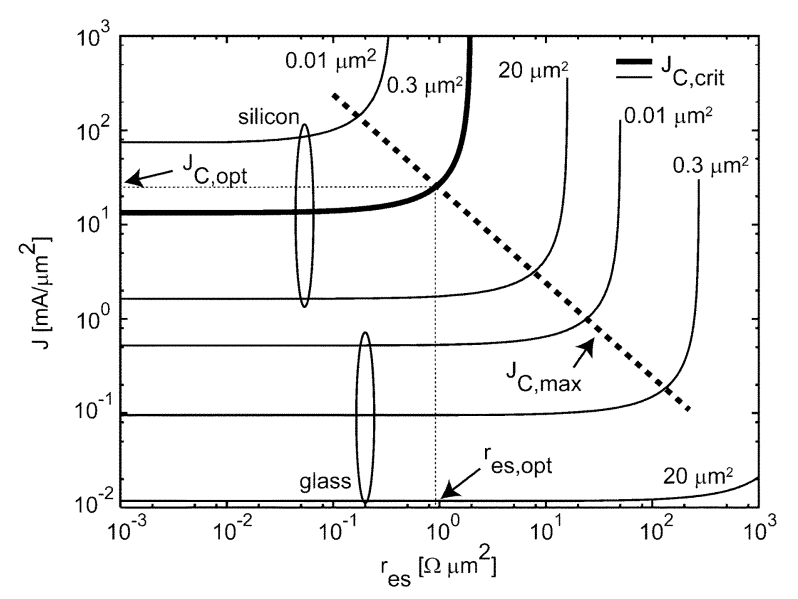

Fig. 7. Current densities $J_{C \text {, max }}$ (dashed line) and $J_{C \text {, crit }}$ (solid lines) versus specific emitter resistivity for three different emitter areas, and for both silicon and glass substrates.

$20 \mathrm{~mA} / \mu \mathrm{m}^{2}$ and $r_{\mathrm{es}}$ of $1.2 \Omega \mu \mathrm{m}^{2}$, (3) and (7) yield $J_{C \text {,crit }}$ (silicon) $=41.5 \mathrm{~mA} / \mu \mathrm{m}^{2}$ and $A_{E \text {,crit }}$ (silicon) $=0.13 \mu \mathrm{m}^{2}$. In Fig. $7 J_{C, \text { crit }}-r_{\text {es }}$ and $J_{C, \max }-r_{\text {es }}$ characteristics defined by (3) and (6) are plotted versus $r_{\mathrm{es}}$. For the bulk-silicon device with $A_{E}=0.3 \mu \mathrm{m}^{2},(8)$ and (9) yield $r_{\text {es,opt }}=1 \Omega \mu \mathrm{m}^{2}$ and $J_{C \text {,opt }}=27.5 \mathrm{~mA} / \mu \mathrm{m}^{2}$. The $350-\mathrm{GHz}$ device has parameters approaching these values. 


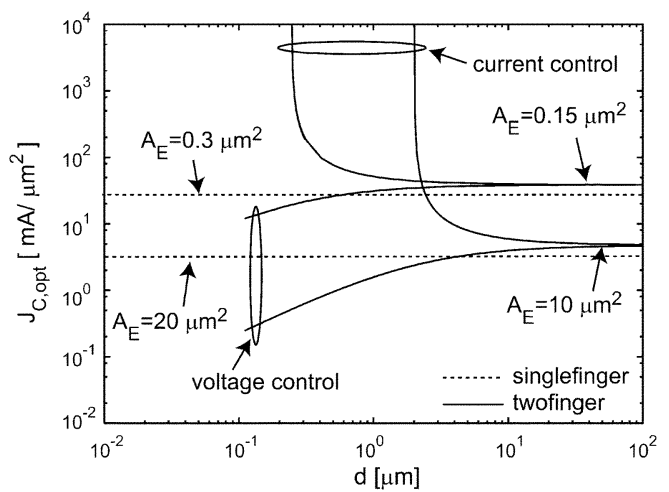

Fig. 8. Calculated optimum current density of two-finger (solid lines) and single-finger (dotted lines) devices. The effect of the finger pitch $d$ on $J_{C \text {,opt }}$ is plotted for two-finger devices in both voltage- and current-controlled biasing conditions.

The mutual thermal coupling $R_{M}$ from (10) is approximated by a point-to-point thermal resistance [14] $R_{M}=1 /\left(2 \pi k_{\text {sub }} d\right)$, where $d$ is the pitch between the neighboring fingers. As an example, single-finger transistors with $A_{E}$ of 20 and $0.3 \mu \mathrm{m}^{2}$ are compared to two-finger devices with $A_{E}$ s equal to $2 \times 10 \mu \mathrm{m}^{2}$ and $2 \times 0.15 \mu \mathrm{m}^{2}$, respectively. The optimum current densities are calculated versus the finger pitch for both the voltage- and current-controlled biasing, and are compared to $J_{C \text {,opt }}$ of the corresponding single-finger transistor in Fig. 8. If a current-controlled biasing is used, splitting a single-finger transistor into a two-finger device results in an increase in the $J_{C \text {,opt }}$ regardless of the value of the pitch. For this type of biasing, bringing fingers closer to each other results in a significant increase of $J_{C \text {,opt }}$, and is also beneficial for reducing the device area. On the other hand, if a voltage-controlled biasing is used, the $J_{C \text {,opt }}$ increases for a large finger pitch, but reducing the distance between the fingers in order to decrease the total area of the device, can result in a decrease of $J_{C \text {,opt }}$, which can eventually become lower than that of the single-finger device.

\section{CONCLUSION}

From the above analysis, it becomes clear that the speed requirements of advanced bipolar transistors is driving the emitter dimensions and resistance into a region where the very high power densities and the significant thermal resistances will lead to significant device self-heating and detrimental electrothermal feedback. These thermal effects set a maximum current for the safe operating region of the device, which can be extremely low for devices isolated on all sides by dielectrics. For more conventional bulk-silicon devices the maximum current is orders higher but the presented theory predicts that the limit on the current density of high-frequency bipolar devices will, before too long, be set by electrothermal effects.

\section{REFERENCES}

[1] H. Nakajima, N. Itoh, K. Inou, T. Iinuma, S. Matsuda, C. Yoshino, Y. Katsumata, and $\mathrm{H}$. Iwai, " $0.5 \mu \mathrm{m}$ silicon bipolar transistor technology for analog applications," in Proc. BCTM, 1994, pp. 213-216.
[2] A. Pruijmboom, D. Szmyd, R. Brock, R. Wall, N. Morris, F. Keng, and F. Jovenin, "QUBiC3: A $0.5 \mu \mathrm{m}$ BiCMOS production technology, with $f_{T}=30 \mathrm{GHz}, f_{\max }=60 \mathrm{GHz}$ and high-quality passive components for wireless telecommunication applications," in Proc. BCTM, 1998, pp. $120-123$.

[3] S. A. S. Onge, D. L. Harame, J. S. Dunn, S. Subbanna, D. C. Ahlgren, G. Freeman, B. Jagannathan, J. Jeng, K. Schonenberg, K. Stein, R. Groves, D. Coolbaugh, N. Feilchenfeld, P. Geiss, M. Gordon, P. Gray, D. Hershberger, S. Kilpatrick, R. Johnson, A. Joseph, L. Lanzerotti, J. Malinowski, B. Orner, and M. Zierak, "A $0.24 \mu \mathrm{m} \mathrm{SiGe} \mathrm{BiCMOS} \mathrm{mixed-}$ signal RF production technology featuring a $47 \mathrm{GHz} f_{T} \mathrm{HBT}$ and 0.18 $\mathrm{m} L_{\text {eff }}$ CMOS," in Proc. BCTM, 1999, pp. 117-120.

[4] D. Szmyd, R. Brock, N. Bell, S. Harker, G. Patrizi, J. Fraser, and R. Dondero, "QUBiC4: A silicon RF-BiCMOS technology for wireless communication ICs," in Proc. BCTM, 2001, pp. 60-63.

[5] K. Oda, E. Ohue, I. Suzumura, R. Hayami, A. Kodama, H. Shimamoto, and K. Washio, "Self-aligned selective-epitaxial-growth $\mathrm{Si}_{1-x-y} \mathrm{Ge}_{x} \mathrm{C}_{y}$ HBT technology featuring 170-GHz $f_{\max }$," in IEDM Tech. Dig., 2001, pp. 332-335.

[6] S. J. Jeng, B. Jagannathan, J.-S. Rieh, J. Johnson, K. T. Schonenberg, D. Greenberg, A. Stricker, H. Chen, M. Khater, D. Ahlgren, G. Freeman, K. Stein, and S. Subbanna, "A $210-\mathrm{GHz} f_{T}$ SiGe HBT with a nonselfaligned structure," IEEE Electron Device Lett., vol. 22, pp. 542-544, Nov. 2001.

[7] B. Jagannathan, M. Khater, F. Pagette, J.-S. Rieh, D. Angell, H. Chen, J. Florkey, F. Golan, D. R. Greenberg, R. Groves, S. J. Jeng, J. Johnson, F. Mengistu, K. T. Schonenberg, C. M. Schnabel, P. Smith, A. Stricker, D. Ahlgren, G. Freeman, K. Stein, and S. Subbanna, "Self-aligned site NPN transistors with $285 \mathrm{GHz} f_{M A X}$ and $207 \mathrm{GHz} f_{T}$ in a manufacturable technology," IEEE Electron Device Lett., vol. 23, pp. 542-544, May 2002.

[8] J.-S. Rieh, B. Jagannathan, H. Chen, K. T. Schonenberg, D. Angell, A. Chinthakindi, J. Florkey, F. Golan, D. Greenberg, S.-J. Jeng, M. Khater, F. Pagette, C. Schnabel, P. Smith, A. Stricker, K. Vaed, R. Volant, D. Ahlgren, and G. Freeman, "SiGe HBTs with cut-off frequency of 350 GHz," in IEDM Tech. Dig., 2002, pp. 771-774.

[9] A. Joseph, P. Geiss, X. Liu, J. Johnson, K. Schonenberg, A. Chakravarti, D. Ahlgren, and J. Dunn, "Emitter resistance improvement in high-performance SiGe HBTs," in Proc. ISTDM, 2003, pp. 53-54.

[10] R. P. Arnold and D. S. Zoroglu, "A quantitative study of emitter ballasting," IEEE Trans. Electron Devices, vol. ED-21, pp. 385-391, July 1974.

[11] R. Dekker, P. Baltus, M. van Deurzen, W. v. d. Einden, H. Maas, and A. Wagemans, "An ultra low-power RF bipolar technology on glass," in IEDM Tech. Dig., 1997, pp. 921-922.

[12] R. Dekker, P. G. M. Baltus, and H. G. R. Maas, "Substrate transfer for RF technologies," IEEE Trans. Electron Devices, vol. 50, pp. 747-757, Mar. 2003.

[13] L. K. Nanver, N. Nenadović, V. d'Alessandro, H. Schellevis, H. W. van Zeijl, R. Dekker, D. B. de Mooij, V. Zieren, and J. W. Slotboom, "A back-wafer contacted silicon-on-glass integrated bipolar process, part I-The conflict electrical versus thermal isolation," IEEE Trans. Electron Devices, vol. 51, pp. 42-50, Jan. 2004.

[14] A. Pacelli, P. Palestri, and M. Mastrapaqua, "Compact modeling of thermal resistance in bipolar transistors on bulk and SOI substrates," IEEE Trans. Electron Devices, vol. 49, pp. 1027-1033, Jun. 2002.

[15] D. T. Zweidinger, S.-G. Lee, and R. M. Fox, "Compact modeling of BJT self-heating in SPICE," IEEE Trans. Computer-Aided Design, vol. 12 , pp. 1368-1375, Sep. 1993.

[16] FemLab Users Guide and Introduction, Comsol AB, Stockholm, Sweden, 2003.

[17] N. Nenadović, V. Cuoco, S. J. C. H. Theeuwen, H. Schellevis, G. Spierings, A. Griffo, M. Pelk, L. K. Nanver, R. F. F. Jos, and J. W. Slotboom, "RF power silicon-on-glass VDMOSFETs," IEEE Electron Device Lett., vol. 25, pp. 424-426, Jun. 2004

[18] R. H. Winkler, "Thermal properties of high-power transistors," IEEE Trans. Electron Devices, vol. ED-14, pp. 260-263, May 1967.

[19] N. Nenadović, V. d'Alessandro, F. Tamigi, A. Rossi, A. Griffo, L. K. Nanver, and J. W. Slotboom, "Thermal instability in two-finger bipolar transistors," in Proc. ESSDERC, 2003, pp. 203-206.

[20] N. Nenadović, V. d'Alessandro, L. K. Nanver, F. Tamigi, N. Rinaldi, and J. W. Slotboom, "A back-wafer contacted silicon-on-glass integrated bipolar process, part II-A novel analysis of thermal breakdown," IEEE Trans. Electron Devices, vol. 51, pp. 51-62, Jan. 2004. 
[21] J. W. Slotboom, "The shrinking bipolar transistor," in Proc. BCTM, 2003, p. i. 16

[22] P. Agarwal, H. G. A. Huizing, and P. H. C. Magneé, "The influence of parasitic resistances on the $f_{T}$ optimization of high-speed SiGe-HBTs," in Proc. ESSDERC, 2003, pp. 291-294.

[23] W. Liu, "Thermal coupling in 2-finger heterojunction bipolar transistors," IEEE Trans. Electron Devices, vol. 42, pp. 1033-1038, Jun. 1995.

[24] R. Dekker and C. E. Timmering, "Copper substrate transfer technologies for silicon RF circuits," in Proc. BCTM, 2003, pp. 31-34.

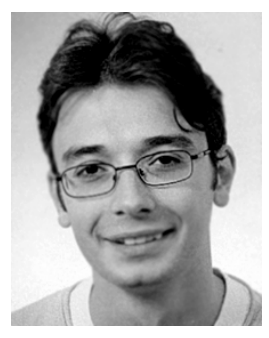

Nebojša Nenadović (S'02) was born in Belgrade, Serbia and Montenegro, on September 13, 1976. $\mathrm{He}$ received the degree (with honors) in electrical engineering from the University of Belgrade, in 2000, and the Ph.D. degree from the Laboratory of Electronic Components, Technology and Materials, Delft Institute of Microelectronics (DIMES), Delft University of Technology, The Netherlands, in 2004, where he is currently a Postdoctoral Research Fellow.

During 1998 and 1999, he was involved in the investigation of transitions in the quantum Hall regime at the Department of Condensed Matter Physics, Weizmann Institute of Science, Israel. His research experience also includes modeling and simulation of optoelectronic devices, in particular quantum-wire lasers, at the Department of Physical Electronics, Faculty of Electrical Engineering, University of Belgrade. His current research interests are design, fabrication, and characterization of BJTs and RF power MOSFETs in silicon and silicon-on-glass technology, and the investigation of electrothermal interactions within semiconductor devices.

Dr. Nenadović received the Best Student Paper Awards for his contributions at the IEEE Topical RF Meeting in 2001, and the IEEE MIEL Conference in 2002.

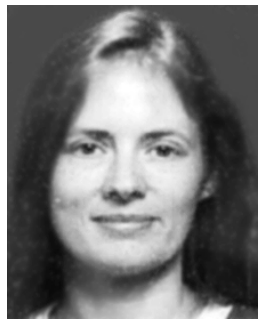

Lis K. Nanver ( $\left.{ }^{\prime} 80-\mathrm{M}^{\prime} 83\right)$ received the M.Sc. degree in physics in 1979 from the University of Aarhus, Aarhus, Denmark. In 1982, she received the Dr.ing. degree from the Ecole Nationale Superieure des Télécommunications, Paris, France, where she worked on the simulation of CCD structures. In 1987, she received the Ph.D. degree from the Delft University of Technology, Delft, The Netherlands, where she developed a medium-frequency BIFET process.

In 1988, she joined the Delft Institute of Microelectronics (DIMES), IC Process Research Sector as Bipolar Process Research Manager. She became Associate Professor and later Professor with the Faculty of Electrical Engineering, Mathematics and Computer Science, Delft University of Technology, detached at the DIMES Technology Center in 1994 and 2001, respectively. Within the Laboratory of Electrical Components, Technology and Materials she manages the research on advanced Si-based devices that is mainly directed toward the optimization and development of high-frequency Si BJT/SiGe HBT devices and the integration of passives. This research involves technologies such as AP/LPCVD epitaxy, dopant activation by excimer laser annealing, and substrate transfer techniques.

Dr. Nanver has served on the committees of the ESSDERC, BCTM, and SBMicro.

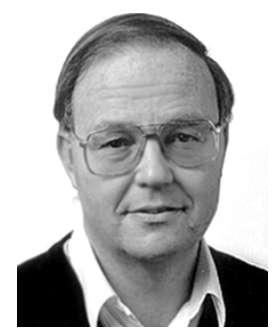

Jan W. Slotboom (M'82) was born in Utrecht, The Netherlands, on December 26, 1942. He received the M.Sc. degree in electrical engineering from the Technical University of Delft, Delft, The Netherlands, in 1966, and the Ph.D. degree from the Technical University of Eindhoven, Eindhoven, The Netherlands, in 1977. His dissertation was on 2-D numerical device simulation of bipolar transistors and pioneering experiments on bandgap narrowing in heavily doped silicon.

In 1967, he joined the Philips Research Laboratories, Eindhoven, where he worked on bipolar device modeling, numerical simulation, and experimental silicon device physics. He was involved in the development of CCD memories for video applications and exploratory research of high-density memories. He has authored and coauthored over 50 papers and holds 14 patents. Since 1994, he has been a Parttime Professor at the Delft Institute of Microelectronics (DIMES), Delft University of Technology. His current research activities are on research of novel silicon devices (e.g., SiGe bipolar transistors), hot-carrier effects, and new memory cells.

Dr. Slotboom was a Member of the Solid-State Devices Subcommittee of the IEDM in 1980, 1983, and 1984, respectively. He was Vice Chairman and Chairman of International Arrangements for Europe of the IEDM. He has served as a Program Subcommittee Member of the IEDM in 1991 and 1992 and of the BCTM'95 Conference. He has been a Member of the Technical Program Committee of the ESSDERC for the last six years. 